\title{
Variations in the immune response to natural Schistosoma mattheei infections in calves born to infected mothers
}

\author{
S. Gabriël ${ }^{\mathrm{a}, \mathrm{d}}$, I.K. Phiri ${ }^{\mathrm{a}}$, G.J. Van Dam ${ }^{\mathrm{b}}$, A.M. Deelder ${ }^{\mathrm{b}}$, \\ L. Duchateau ${ }^{\mathrm{c}}, \mathrm{J}$. Vercruysse ${ }^{\mathrm{d}, *}$ \\ ${ }^{a}$ Department of Clinical Studies, School of Veterinary Medicine, University of Zambia, \\ Box 32379, Lusaka, Zambia \\ ${ }^{\mathrm{b}}$ Department of Parasitology, Leiden University Medical Centre, \\ P.O. Box 9600, 2300 RC Leiden, The Netherlands \\ c Department of Physiology, Biochemistry and Biometrics, Ghent University, \\ Salisburylaan 133, 9820 Merelbeke, Belgium \\ d Department of Virology, Parasitology and Immunology, Faculty of Veterinary Medicine, \\ Ghent University, Salisburylaan 133, 9820 Merelbeke, Belgium
}

Received 3 June 2003; received in revised form 28 October 2003; accepted 4 November 2003

\begin{abstract}
During previous work Schistosoma antibodies and circulating antigens were detected at birth in the serum from some calves born to Schistosoma mattheei infected mothers. The objectives of the present survey were: (1) to investigate the proportion of calves, born to cows infected with $S$. mattheei, which have specific antibodies and circulating schistosome antigens present in their serum at birth and (2) to investigate whether the presence or absence of these specific antibodies and/or circulating antigens at birth may affect the pattern of a natural $S$. mattheei infection in calves from 4 to 5 months of age, when the colostral antibodies are thought to be of negligible importance. A total of 28 calves born to infected mothers were randomly selected. Faeces, serum and colostrum samples were collected from the cows at calving, serum samples were collected from the calves at birth (day 0 ), after intake of colostrum (day 1) and monthly thereafter up to the age of 10 months. Both serum and colostrum samples were analysed for $\operatorname{IgG}_{\mathrm{H}+\mathrm{L}}$ against SWAP matthee $i$ and schistosome circulating anodic antigen (CAA) levels. The calves were exposed to a natural challenge from the age of 4-5 months. Faecal samples were collected from the calves monthly, starting at an age of 5 months up to 10 months, and were examined for faecal egg counts. Nine (group 1) out of the 28 calves were found to have specific antibodies in their serum at birth, in 5 of them CAA levels were also detected. In the other 19 calves (group 2) no $\mathrm{IgG}_{\mathrm{H}+\mathrm{L}}$ or CAA were detected. At the end of the study faecal egg counts and CAA levels were significantly lower in calves from group 1 compared
\end{abstract}

\footnotetext{
* Corresponding author. Tel.: +32-9-2647390; fax: +32-9-2647496.

E-mail address: jozef.vercruysse@ugent.be (J. Vercruysse).
} 
to group 2. Results confirm earlier work that specific antibodies and circulating antigens may be present in serum from calves at birth, and show that these calves have lower faecal egg counts and CAA levels after exposure to a natural challenge.

(C) 2003 Elsevier B.V. All rights reserved.

Keywords: Schistosoma mattheei; Cattle-trematoda; Transplacental transfer; Schistosomal circulating anodic antigen; Immunoglobulins

\section{Introduction}

In schistosome endemic areas calves can become infected with Schistosoma mattheei very early in life. Calves are the most susceptible age group to infection and pass large numbers of viable eggs in their faeces. Development of acquired resistance observed mainly as a reduction in fecundity of the female worms around the age of 18 months has been described previously (Bushara et al. 1980, 1983a,b; Majid et al., 1980; Kassuku et al., 1986; De Bont et al., 1995). This acquired resistance appears to be immunologically induced, but almost nothing is known on the actual mechanism(s) of protection. Results from Bushara's work (1994) provide evidence that fecundity suppression following acquired resistance to $S$. bovis is due at least in part to serum born factors: $S$. bovis infected calves injected with immune serum from donors given a single experimental infection, showed reduced egg counts and number of worms in comparison with control animals.

Within a same herd, large individual variations are observed in egg output of calves (De Bont et al., 1995). Variations in susceptibility to a first natural challenge could be explained by the differences in the type and quality of the passive immunity received from the cow. A recent study (Gabriel et al., 2002a) indicated that the intake of colostrum from $S$. mattheei infected cows partially protected young calves against an early experimental $S$. mattheei infection, i.e. when antibody levels were still elevated, expressed as a significant reduction of $42 \%$ in total worm and tissue egg counts, and a reduction of $25 \%$ in faecal egg counts. It was also determined that the levels of specific antibodies against SWAP (soluble adult worm antigens preparation) mattheei in the serum from young calves received from colostrum from infected mothers are still elevated at the age of 1 month but decrease to negligible levels at 2-3 months of age.

Gabriel et al. (2002b) also detected specific antibodies and circulating schistosome antigens at birth-before intake of colostrums - in the sera of four out of six calves born to infected mothers. As antibody passage is not possible through the normal ruminant placenta (Kruse, 1983) the presence of the specific antibodies and antigens was explained by a transplacental transfer of schistosome circulating antigens causing an intrauterine antigenic stimulation, resulting in the antibody production by the foetus (Gabriel et al., 2002b). Studies from King et al. (1998) indicated that the human foetus can be sensitised to produce helminth-specific B cells and that neonatal B cells are capable of antibody production.

It is yet unclear whether this possible intrauterine priming could also influence the immune reaction towards (and protection against) infections. 
The objectives of the present survey were: (1) to investigate the proportion of calves born to cows infected with $S$. mattheei, which has specific antibodies and circulating schistosome antigens (CAA) present in their serum at birth and (2) to investigate whether the presence of these specific antibodies and/or circulating antigens at birth may influence the pattern of a natural $S$. mattheei infection in calves from 4 to 5 months of age, when the colostral antibodies are thought to be of negligible importance.

\section{Materials and methods}

\subsection{Study site and animals}

The study was carried out on an extensive beef farm in Chisamba, in the Central Province of Zambia. The 8100 ha estate is largely covered by open indigenous woodland where the herd of 1500 Simmental-Boran crossbred animals is allowed to graze day and night. A total of 28 cows and their new-born calves (June 2001) were randomly selected for the present survey. All mothers carried an active infection as determined by detection of eggs and/or miracidia in the faeces and/or specific antibodies against SWAP mattheei and circulating schistosome antigens in the serum. The survey was conducted from birth up to the age of 10 months.

\subsection{Experimental design}

At calving faeces, serum and colostrum samples were collected from the 28 cows. Serum was collected from the calves before (day 0) intake of colostrum and according to the presence or lack of specific antibodies against SWAP mattheei at birth the calves were divided in groups 1 and 2, respectively. Serum samples were also collected $24 \mathrm{~h}$ after intake of colostrum (day 1) and then monthly up to the age of 10 months. The calves were born in a paddock without transmission sites (drinking water was available from a borehole) and were moved to a grazing area with infected Schistosoma transmission sites at the age of about 4-5 months. All calves remained in contact with infected transmission sites during the rest of the 10 months observation period. Faecal samples were collected monthly from the calves, starting at an age of 4 months up to 10 months.

Faecal samples were examined for faecal egg counts and the sera and colostrums were analysed for specific $\mathrm{IgG}_{\mathrm{H}+\mathrm{L}}$ levels. CAA levels were determined in the serum and colostrums from the mothers, and in the serum of the calves on days 0, 1, 180, 240, 270 and 300.

\subsection{Parasitological techniques}

To determine the faecal egg counts (EPG) a modification of the concentration technique of Lawrence (1970) (1 egg = 10 epg) according to De Bont et al. (1991, 1995) was used, with a sensitivity of 2.5 instead of 10 eggs per gram. 


\subsection{Enzyme-linked immunosorbent assay}

The enzyme-linked immunosorbent assay was used to determine Schistosoma-specific $\mathrm{IgG}_{\mathrm{H}+\mathrm{L}}$ levels against SWAP matthee $i$ in the serum and in the colostrum samples according to Gabriel et al. (2002a) using Soluble Adult Worm antigens Preparation (SWAP, Viana da Costa et al., 1998) mattheei Ag (=total S. mattheei antigen). Delta optical densities ( $\Delta \mathrm{OD})$ were calculated according to the formula:

$$
\Delta \mathrm{OD}=\mathrm{OD} \mathrm{x}-\mathrm{OD} \text { neg }
$$

where OD $x$ is the individual OD value of the test serum, and OD neg the OD of a known negative sample.

\subsection{Circulating anodic antigen (CAA) determination}

CAA levels $(\mathrm{pg} / \mathrm{ml})$ were determined by a monoclonal antibody-based antigen-capture ELISA as described by Polman et al. (2000) with minor modifications. Forty pg CAA/ml was taken as cut-off level giving a specificity of $98 \%$.

\subsection{Statistical analysis}

Assuming a normal distribution, a mixed model with calves as random effect was fitted to evaluate the differences in immunoglobulin and CAA levels in the sera between the two groups. As epg levels could not be assumed to be normally distributed, the analysis for this outcome variable was based on the nonparametric Wilcoxon-Mann-Whitney test stratified for time (using measurements at days 240, 270 and 300 after birth). Furthermore, $\mathrm{IgG}_{\mathrm{H}+\mathrm{L}}$ and CAA levels were compared between the two groups of infected mothers based on a $t$-test and the epg levels were compared based on the Wilcoxon-Mann-Whitney test.

\section{Results}

\subsection{Cows}

The median faecal egg counts and the mean levels of specific $\operatorname{IgG}_{\mathrm{H}+\mathrm{L}}$ against SWAP matthee $i$ and CAA are presented in Table 1. There were no significant differences in epg, $\mathrm{IgG}_{\mathrm{H}+\mathrm{L}}$ or CAA levels (serum and colostrums) between groups 1 and 2 .

\subsection{Calves at birth and 1 day after intake of colostrum}

Of the 28 examined calves, 9 calves showed elevated $\operatorname{IgG}_{\mathrm{H}+\mathrm{L}}$ levels against SWAP mattheei in their serum at birth (group 1) while no specific antibodies were detected at birth in 19 calves (group 2) (Table 1). From the 9 calves with elevated $\mathrm{IgG}_{\mathrm{H}+\mathrm{L}}$ levels 5 showed elevated CAA levels (ranging from 390 to $5460 \mathrm{pg} / \mathrm{ml}$ ), in the other 4 no CAA was 
Table 1

Mean values of $\mathrm{IgG}_{\mathrm{H}+\mathrm{L}}$ levels $(\triangle \mathrm{OD})$ and CAA levels $(\mathrm{pg} / \mathrm{ml})$ in the serum and colostrum from the mothers, and in the serum from the calves of group $1^{\mathrm{a}}$ and of EPG group 2 on day 0 and day 1

\begin{tabular}{|c|c|c|c|c|c|c|}
\hline & \multicolumn{3}{|l|}{ Group 1} & \multicolumn{3}{|l|}{ Group 2} \\
\hline & $\operatorname{IgG}_{\mathrm{H}+\mathrm{L}}$ & CAA & $\mathrm{EPG}^{*}$ & $\mathrm{IgG}_{\mathrm{H}+\mathrm{L}}$ & CAA & $\mathrm{EPG}^{*}$ \\
\hline \multicolumn{7}{|l|}{ Mothers } \\
\hline Serum & $0.804(0.403-1.515)$ & $149586(102-629340)$ & $12.5(0-30)$ & $0.767(0.219-1.855)$ & $153226(707-583710)$ & $5(0-50)$ \\
\hline Colostrum & $1.94(1.556-2.263)$ & 9254 (1076-32355) & & $1.62(0.691-2.91)$ & $9712(947-43246)$ & \\
\hline \multicolumn{7}{|l|}{ Calves } \\
\hline Day 0 & $0.839^{\mathrm{b}}(0.23-1.988)$ & $1232^{\mathrm{b}}(0-5460)$ & & $0^{\mathrm{b}}(0-0)$ & $0^{\mathrm{b}}(0-0)$ & \\
\hline
\end{tabular}

* Median faecal egg counts of the mothers at birth

${ }^{\text {a }}$ See text for definition of groups 1 and 2 .

b Significant differences. 


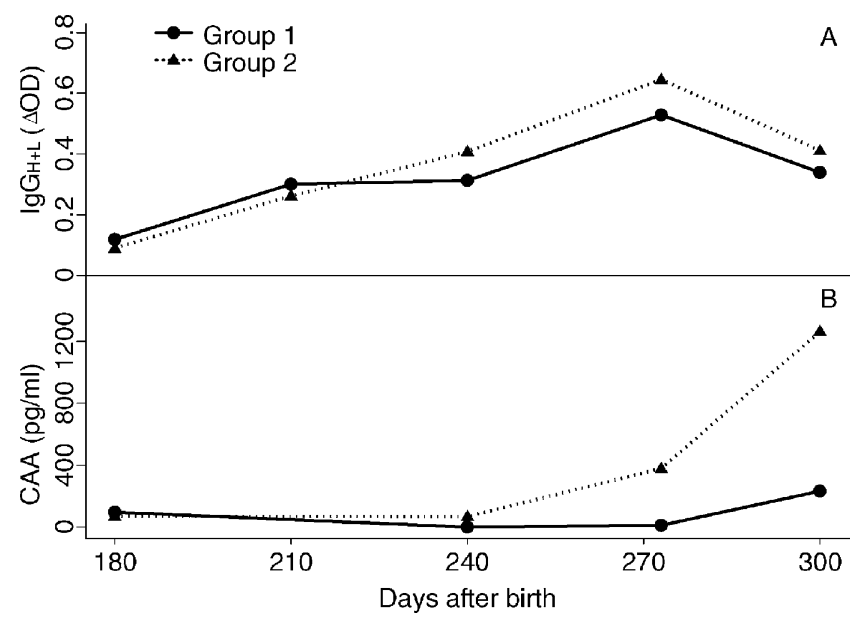

Fig. 1. (A) Mean $\operatorname{IgG}_{\mathrm{H}+\mathrm{L}}$ levels against SWAP mattheei $(\Delta \mathrm{OD})$ in the sera from calves naturally infected with $S$. mattheei around the age of 4-5 months. Group 1: calves with specific antibodies detected at birth. Group 2: calves with no specific antibodies detected at birth. (B) Mean CAA levels (pg/ml) in the sera from calves naturally infected with $S$. mattheei around the age of 4-5 months. Group 1: calves with specific antibodies detected at birth. Group 2: calves with no specific antibodies detected at birth.

detected. In the serum from the calves from group 2 no CAA was detectable. After intake of colostrum the $\mathrm{IgG}_{\mathrm{H}+\mathrm{L}}$ and CAA levels increased in both groups and only the CAA levels of group 2 remained significantly lower compared to group 1 (Table 1).

\subsection{Calves until the age of 10 months}

Both $\mathrm{IgG}_{\mathrm{H}+\mathrm{L}}$ and CAA levels decreased after day 1 towards day 180 . The $\operatorname{IgG}_{\mathrm{H}+\mathrm{L}}$ levels for both groups 1 and 2 followed similar patterns from day 180 (after the natural challenge) until day 300 with a maximum mean value on day 270 of $0.529 \Delta \mathrm{OD}$ and $0.646 \Delta \mathrm{OD}$ for groups 1 and 2, respectively (Fig. 1A). There were no significant differences between the two groups from day 180 to day 300. The CAA levels increased from day 240 until day 300 . The increase was minor in group 1 and distinct in group 2 with at day 300 CAA values of 233 and $1265 \mathrm{pg} / \mathrm{ml}$, respectively (Fig. 1B). The CAA levels in group 1 were significantly lower than in group $2(P=0.05)$ from day 240 to day 300. Fig. 2 shows the median faecal egg counts of the two groups of calves. Excretion started at the age of 6 months for 2 animals from group 1 and for 1 animal in group 2. The median faecal egg counts remained low in the two groups until the age of 7 months. Egg counts of group 1 were significantly lower compared to group 2 from day 240 to day $300(P=0.0083)$.

\section{Discussion}

During a previous study specific antibodies against SWAP mattheei and levels of schistosome CAA were observed in four of six calves born to $S$. mattheei infected cows (Gabriel 


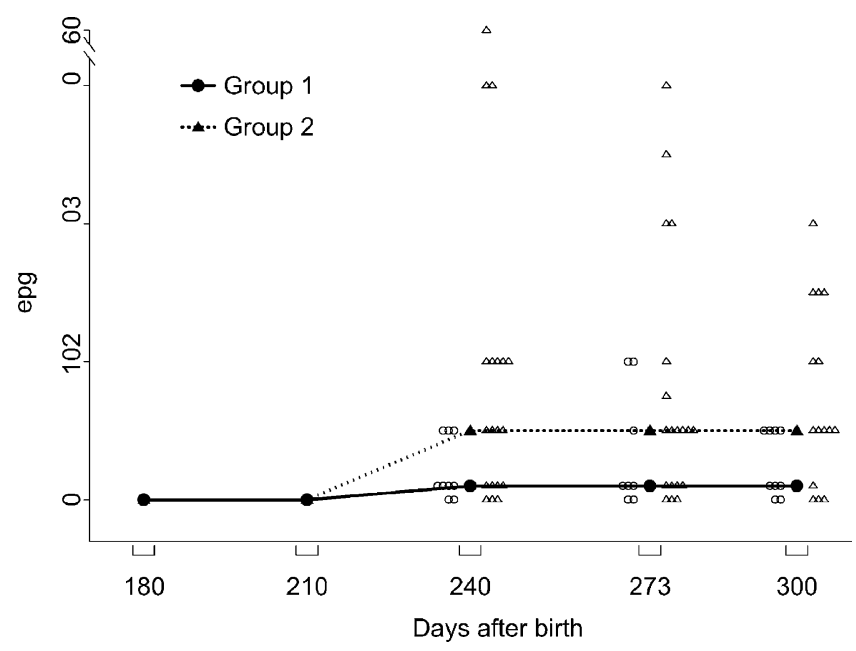

Fig. 2. Median faecal egg counts from Zambian calves infected naturally with $S$. mattheei around 4-5 months of age. Open symbols represent epg values for individual calves. Group 1: calves with specific antibodies. Group 2: calves with no specific antibodies detected at birth.

et al., 2002b). In this study 9 of 28 calves born to infected mothers were observed to have specific antibodies at birth. The fact that not all of them had CAA levels in their serum as well could be due to biological variations in, e.g. antigen clearance mechanisms, however, this requests further research. The presence of specific antibodies and circulating antigens in the calves born to infected mothers at birth in the first study was thought to be caused by a transplacental antigen transfer followed by an intra-uterine priming (Gabriel et al., 2002b). Other possible hypotheses for their presence, such as the transplacental transfer of worms (Johansen et al., 2002), or the transfer of maternal antibodies have been discussed before and found to be rather unlikely (Gabriel et al., 2002b). However, need arises to investigate further the origin and type of these specific antibodies and antigens, as well as the causes triggering the transfer.

On day 1 , after intake of colostrum, CAA levels increased for both groups. This could be indicative for a good intestinal absorption of the circulating schistosome antigens present in the colostrum, in contrast with earlier work (Gabriel et al., 2002b) where a minor absorption was noticed. The significantly higher CAA levels recorded on day 1 for group 1 were expected as levels were already elevated before the intestinal absorption of the colostrum started.

During previous work it was determined that at the age of 1 month the level of specific maternal antibodies in the serum from the calf, received through the colostrum, was still high, and a partial protection against an early $S$. mattheei infection was observed (Gabriel et al., 2002a,b). In the present study, at the age of 5 months, when the calves received a natural challenge, the levels of specific $\mathrm{IgG}_{\mathrm{H}+\mathrm{L}}$ were very low (mean value of $0.13 \Delta \mathrm{OD}$ ), suggesting that the specific antibodies received from the mother declined to insignificant levels and therefore might be of minor importance in protection against subsequent infection. The significant lower faecal egg counts and CAA levels, together with the $39 \%$ lower mean 
CCA (Circulating Cathodic Antigen, a different parasite excretory antigen) levels (data not shown) in the 10-month-old calves, that had antibodies at birth (group 1) are indicative that the suggested intra-uterine priming (Gabriel et al., 2002b) could modify a subsequent natural infection. Other studies determined that prenatal exposure to schistosome antigen could alter immune response towards later contact with schistosome eggs expressed by a significant increase or decrease in granuloma size, however, effects on infection patterns were not considered (Lewert and Mandlowitz, 1969; Hang et al., 1974; Camus et al., 1976). Novato-silva et al. (1992) determined proliferative responses by the cord blood mononuclear cells of many neonates born to infected mothers after stimulation with schistosomal antigens, which indicated a cell-mediated sensitisation in utero. Silverstein et al. (1963) demonstrated that the foetal lamb in utero is capable of responding to the intra-foetal injection of antigen with the production of circulating antibodies, in rather substantial amounts. They also determined differences in response depending on the type of antigen injected.

Results from this study confirm earlier work with the detection of specific antibodies in the serum from new-born calves. This study also indicates a possible protective effect of the suggested intra-uterine priming on infection levels after a natural infection.

\section{Acknowledgements}

This work was supported by the Flemish Inter University Council (Belgium). The authors wish to thank Sable Farms and Dr. and Mrs. Robey for their cooperation in the survey, professor Goddeeris, Department of Virology, Immunology, Parasitology, Faculty of Veterinary Medicine, Belgium and Dr. De Bont, International AIDS Vaccine Initiative, New York, for their attributions to the discussion, the technicians of the Department of Clinical Studies, School of Veterinary Medicine, Zambia, and Mrs. D. Kornelis from the Department of Parasitology, LUMC, Leiden, The Netherlands for their excellent technical assistance.

\section{References}

Bushara, H.O., Majid, A.A., Saad, A.M., Hussein, M.F., Taylor, M.G., Dargie, J.D., Marshall, T.F., De, C., Nelson, G.S., 1980. Observations on cattle schistosomiasis in the Sudan, a study on comparative medicine. II. Experimental demonstration of naturally acquired resistance to Schistosoma bovis. Am. J. Trop. Med. Hyg. 29, $442-451$.

Bushara, H.O., Gameel, A.A., Majid, B.Y.A., Khitma, I., Haroun, E.M., Karib, E.A., Hussein, M.F., Taylor, M.G., 1983a. Observations on cattle schistosomiasis in the Sudan, a study in comparative medicine. VI. Demonstration of resistance to Schistosoma bovis challenge after single exposure to normal cercariae or to transplanted adult worms. Am. J. Trop. Med. Hyg. 32, 1375-1380.

Bushara, H.O., Hussein, M.F., Majid, M.A., Musa, B.E.H., Taylor, M.G., 1983b. Observations on cattle schistosomiasis in the Sudan, a study in comparative medicine. IV. Preliminary observations on the mechanism of naturally acquired resistance. Am. J. Trop. Med. Hyg. 32, 1065-1070.

Bushara, H.O., Omer, O.H., Malik, K.H.E., Taylor, M.G., 1994. The effect of multiple transfers of immune serum on maturing Schistosoma bovis infections in calves. Parasitol. Res. 80, 198-202.

Camus, D., Carlier, Y., Bina, J.C., Borojevic, R., Prata, A., Capron, A., 1976. Sensitization to Schistosoma mansoni antigen in uninfected children born to infected mothers. J. Infect. Dis. 134, 405-408.

De Bont, J., Vercruysse, J., Sabbe, F., Southgate, V.R., Rollinson, D., 1995. Schistosoma mattheei infections in cattle: changes associated with season and age. Vet. Parasitol. 57, 299-307. 
De Bont, J., Vercruysse, J., Van Aken, D., Southgate, V.R., Rollinson, D., Moncrieff, C., 1991. The epidemiology of Schistosoma spindale Montgomery, 1906 in cattle in Sri Lanka. Parasitology 102, 237-241.

Gabriel, S., De Bont, J., Phiri, I.K., Masuku, M., Riveau, G., Schacht, A.M., Billiouw, M., Vercruysse, J., $2002 \mathrm{a}$. The influence of colostrum on early Schistosoma mattheei infections in calves. Parasitology 125, 537-544.

Gabriel, S., De Bont, J., Phiri, I.K., Masuku, M., Riveau, G., Schacht, A.M., Deelder, A.M., Van Dam, G.J., Vercruysse, J., 2002b. Transplacental transfer of schistosomal circulating anodic antigens in cows. Parasite Immunol. 124, 521-525.

Hang, L.M., Boros, D.L., Warren, K.S., 1974. Induction of immunological hyporesponsiveness to granulomatous hypersensitivity in Schistosoma mansoni infection. J. Infect. Dis. 130, 515-522.

Johansen, M.V., Iburg, G.T., Monrad, J., Ørnberg, N., 2002. Congenital infection with Schistosoma japonicum but not with Schistosoma bovis in sheep. J. Parasitol. 88, 414-415.

Kassuku, A.A., Christensen, N.Ø., Monrad, J., Nansen, P., Knudsen, J., 1986. Epidemiological studies on Schistosoma bovis in iringa region. Tanzania Acta Trop. 43, 153-163.

King, C.L., Malhotra, I., Mungai, P., Wamachi, A., Kioko, J., Ouma, J.H., Kazura, J.W., 1998. B cell sensitization to helminthic infection develops in utero in humans. J. Immunol. 160, 3578-3584.

Kruse, P.E., 1983. The importance of colostral immunoglobulins and their absorption from the intestine of the newborn animals. Ann. Rech. Vet. 14, 349-353.

Lawrence, J.A., 1970. Examination of ruminant faeces for schistosome eggs. Rhod. Vet. J. 1, 49-52.

Lewert, R.M., Mandlowitz, S., 1969. Schistosomiasis: prenatal induction of tolerance to antigens. Nature 224 , 1029-1030.

Majid, A.A., Marchall, T.F.deC., Hussein, M.F., Bushara, H.O., Taylor, M.G., Nelson, G.S., Dargie, J.D., 1980. Observations on cattle schistosomiasis in the Sudan, a study on comparative medicine. I. Epizootical observations on Schistosoma bovis in the White Nile Province. Am. J. Trop. Med. Hyg. 29, 435-441.

Novato-silva, E., Gazzinelli, G., Colley, D.G., 1992. Immune responses during human Schistosomiasis mansoni. XVIII. Immunologic status of pregnant women and their neonates. Scand. J. Immunol. 35, 429-437.

Polman, K., Diakhate, M.M., Engels, D., Nahimana, S., Van Dam, G.J., Falcao Ferreira, S.T., Deelder, A.M., Gryseels, B., 2000. Specificity of circulating antigen detection in Schistosomiasis mansoni in Senegal and Burundi. Trop. Med. Int. Health 5, 534-537.

Silverstein, A.M., Uhr, J.W., Kraner, K.L., Lukes, R.J., 1963. Fetal response to antigenic stimulus. II. Antibody production by the fetal lamb. J. Exp. Med. 117, 799-812.

Viana da Costa, A., Gaubert, S., Fontaine, J., Lafitte, S., Seixas, A., De Lourdes Sapaio Silva, M., Capron, A., Grzych, J.M., 1998. Murine Schistosoma bovis infection: analysis of parasitic and immune parameters. Parasite Immunol. 20, 143-153. 